\title{
An AHP Fuzzy Approach on Outcome-Based Postgraduate Education Quality Evaluation System
}

\author{
Haiying Ma* \\ School of Economics, Northwest University for Nationalities Lanzhou (730124), P.R.China, \\ Ixmahaiying8888@163.com
}

Keywords: Postgraduate; Education quality; Evaluation; Results oriented

\begin{abstract}
In this paper, we analyze outcome-based postgraduate education quality evaluation system. The analysis is conducted by means of an AHP-based Fuzzy Approach which builds a system analysis technique for multiple criteria decision making, and by four aspects: evaluation of employing unit, the government assessment, graduates evaluation and school. The result shows the new system offers the quality of postgraduate education requirement result between employers and feature of the postgraduate student quality. And it is proved that this is an effective tool for high-level academic intuitions and movement to evaluate the quality of postgraduate education with scientific, rational, workable, objective result.
\end{abstract}

\section{Introduction}

Along with the fast economic development in China, the comprehensive national strength and international competitiveness constantly increase the demand for talents. The laborer should constantly improve their own quality level. Countries all over the world pay more and more attention to the development of national education, and the development of postgraduate education is related to the development of its economy. So the scholars and experts in the 1980s carried out the study of graduate education. But due to the different higher education management system, the graduate education quality evaluation in the process of the discussion and research still presents a different point of view and focus. Many scholars have published a large number of papers on the quality of postgraduate education to form their own evaluation system.

Foreign research on the quality of graduate education started in the early of the 1980s. many scholars put research focus on the quality of postgraduate education research, Tony(1993), John And Tarla (2000) and David (2001) argued to improve the quality of graduate education, but the specialized study of interdisciplinary postgraduate education quality evaluation is still less, Tony Becher (2006). Xiong (2001) presented that the purpose of the research of postgraduate education quality is to improve the quality of education, it need to establish a master graduate student teaching evaluation system. The system must meet the needs of social development formed a set of internal and external combination. Pan (2004) pointed out that in terms of the rating system and guarantee the education quality, less common abstraction, quantifiable indicators, indicators of such problems was incomplete. Index system of multifarious, index set lacking of pertinence and operability is poor. Uneven level design and the index can not cover all the disciplines and professional to determine the index weight of subjectivity. Xiao (2009) by social evaluation way, the higher education of China should speed up to establish a quality management system. All the process of education, teaching, course setting and training related ability of practice are interrelated, and the final result we expect to achieve learning. Oriented education based on the results of theory focuses on the following five: one is through education enables the students finally to achieve what the results, the result of this including the knowledge, quality, moral character and ability, etc; Second, why do we need to learn these knowledge: the third is how to accurately obtain the students learning results; Four is how to help students achieve our scheduled learning results; and the last is how to ensure that students can get the results of the study (knowledge, quality, moral character and ability, etc.) therefore, the author set up four evaluation system from the perspective of social dimensions for the primary indicators and the 
corresponding 14 secondary indicators will be examined The rest of this paper is organized as follows: Section 2 describes the methodology and AHP-based Fuzzy model. Section 3 introduces basic principles and structure of index system for outcome-based postgraduate education quality evaluation system. Evaluation process and empirical analysis is discussed in Section 4. Finally, Section 5 concludes the paper.

\section{Methodology and AHP-based Fuzzy Model}

Analytical hierarchy process (AHP) is a system analysis technique for multiple criteria decision making (MCDM) developed by Saaty in the early 1970s. It has been applied in many fields such as engineering, economy, military, politics and management. It stresses the importance of the intuitive judgments of a decision-maker as well as the consistency of the comparison of alternatives in the decision-making process. The method was a multiple decision-making method which combined qualitative with quantitative analysis. It could effectively analyze the non-sequential relationship between levels of target criteria system is as follows:

1) Establishing the index system;

2) Constructing the judgment matrix and single permutation of layer. The indexes of the same level are compared one by one to create judgment matrix. For example, if the elements of layer $A$ have relation to the elements of lower layers $B_{1}, B_{2}, \cdots, B_{n}$, the judgment matrix is shown in Table 1 .

Table 1 Judgment matrix of the significant extent of the indexes in layer $B$

\begin{tabular}{llllll}
\hline$A$ & $B_{1}$ & $B_{2}$ & $\cdots$ & $B_{n}$ & $W_{i}$ \\
\hline$B_{1}$ & $B_{11}$ & $B_{12}$ & $\cdots$ & $B_{1 n}$ & $W_{1}$ \\
$B_{2}$ & $B_{21}$ & $B_{22}$ & $\cdots$ & $B_{2 n}$ & $W_{2}$ \\
$\vdots$ & $\vdots$ & $\vdots$ & $\vdots$ & $\vdots$ & $\vdots$ \\
$B_{n}$ & $B_{n 1}$ & $B_{n 2}$ & $\cdots$ & $B_{n n}$ & $W_{n}$ \\
\hline
\end{tabular}

In the above matrix, $b_{i j}$ shows the significance extent of corresponding $B_{i}$ and $B_{j}$, which takes 1,2 , $3 \ldots, 9$ and their reciprocals as its value. When $b_{i j}$ equals to 1 , it shows that $B_{i}$ and $B_{j}$ are same significance; when $b_{i j}$ equals to 3 , it shows that $B_{i}$ is a little more significant than $B_{j}$; when $b_{i j}$ equals to 5 , it shows that $B_{i}$ is more significant than $B_{j}$; when $b_{i j}$ equals to 7, it shows that $B_{i}$ is much more significant than $B_{j}$; when $b_{i j}$ equals to 9 , it shows that $B_{i}$ is extremely more significant than $B_{j}$; when $b_{i j}$ is $2,4,6$ or 8 , it shows that the significance is between above adjacent values; and the corresponding reciprocal show the insignificance extent.

3 ) Figuring out the eigenvector $\left(W_{i}\right)$, which shows the significance extent of each element in the same layer of judgment matrix, and the biggest eigenvalue $\max \lambda \max$, the equation is:

$$
\lambda_{\max }=\sum_{i=1}\left(A W_{i}\right) / n W_{i}
$$

4) Making statistical test about uniformity. The value of $C I$ is an index evaluating the departure of judgment matrix from uniformity. The equation is:

$C I=\left(\lambda_{\max }-n\right) /(n-1)$

$R I$ is the index of average random uniformity that judges uniformity (the value can be gotten in the statistics table). The proportion of the random uniformity is $C R$, which can be calculated by:

$C R=C I / R I$

If $C R<0.1$, it shows that judgment matrix has a uniformity. Otherwise, the judgment matrix must be adjusted.

5) Sequencing the all layers. This step is to sort the values of relative importance of the layer $C$. 
6) Grading of single element and comprehensive evaluation. Single element grading is to disposal single element as a standardized value through dividing each grade by its accumulation. The comprehensive evaluation $\left(P_{i}\right)$ is to multiply the elevated value of single element multiplied by its weight, then to sum those values to get $P_{i}$, and to compare the results $P_{i}$, and finally to confirm their grades.

$$
P_{i}=\sum_{j=1}^{n} C_{i j} \times E_{i j}
$$

In the above expression, $C_{i j}$ is the standardized data; $E_{i j}$ is weight $\left(W_{i}\right)$ in the aim layer $j, \mathrm{n}$ is the number of the aimed layer.

\section{Establishment of the Index System}

Basic Principles for an Index System. In order to evaluate the integration of OBE construction for postgraduate education quality evaluation system, an appropriate index system is needed to evaluate the complicated objection. The index system should be diversity and multi-dimensional. The indexes must also have mutual constraint and influence each other. The integration degree of OBE construction for postgraduate education quality evaluation system is affected by both internal factors and external factors. Internal factors refer to the inherent factors in the construction mechanism in process of OBE construction for postgraduate education quality evaluation system. The index system should be easy to implement and also all-inclusive to reflect all involved aspects in order to achieve accurate assessment outcomes. A suitable compromise of complexity should be considered to guarantee both practicability and desirable accuracy.

Structure the Outcome-Based Education Evaluation Index System. The OBE construction for postgraduate education quality evaluation system should be established taking the scientific outlook on development as guidance and paying special attention to evaluation of employing unit, the government assessment, graduates evaluation and school. The system requires multi-factors and multi-levels. Based on the connotation of OBE and related literatures, the author initially selected 14 indicators as the preliminary evaluation index system from four aspects, namely "Evaluation of employing unit", "The government assessment" and "Graduates evaluation", "schools". The theoretical evaluation model is shown in Figure1

Table 2 Evaluation index system (layer A-C) of OBE construction for postgraduate education quality evaluation system

\begin{tabular}{|c|c|c|}
\hline Aim layer & Guideline layer A & Sub-guideline layer B \\
\hline \multirow{14}{*}{ 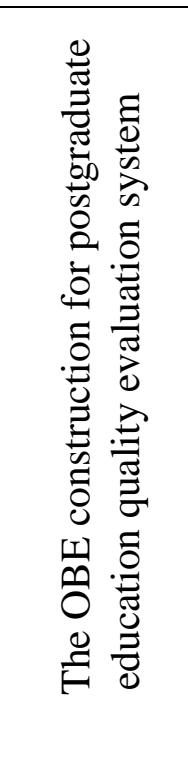 } & \multirow{4}{*}{$\begin{array}{l}\text { A1: Evaluation } \\
\text { of employing unit }\end{array}$} & B1: professional skills \\
\hline & & B2: general skills \\
\hline & & B3: post competency force \\
\hline & & B4:professional quality \\
\hline & \multirow{4}{*}{$\begin{array}{l}\text { A2:The } \\
\text { government } \\
\text { assessment }\end{array}$} & B5: employment rate \\
\hline & & B6: professing rate \\
\hline & & B7: employment quality \\
\hline & & B8: employment structure \\
\hline & \multirow{4}{*}{$\begin{array}{l}\text { A3:Graduates } \\
\text { evaluation }\end{array}$} & B9: scientific research achievements \\
\hline & & B10: recognition to the school \\
\hline & & B11: learning to work contribution rate \\
\hline & & B12: average wage \\
\hline & \multirow{2}{*}{ A4:Schools } & B13: entrepreneurship rate \\
\hline & & B14: comprehensive strength \\
\hline
\end{tabular}




\section{Evaluation Process and Empirical Analysis}

Evaluation Process. On the basis of the evaluation index system of integration of OBE postgraduate education quality evaluation system, the evaluation indexes are divided into four layers according to AHP, the relations among layers and factors are lined out and comparing judgment matrixes, which are composed of the ratios of comparative importance of every pair of factors, and are determined by using experts method. For layer A, the matrix is as follows.

$$
A=\left[\begin{array}{cccc}
1 & 2 & 3 & 4 \\
1 / 2 & 1 & 2 & 3 \\
1 / 3 & 1 / 2 & 1 & 2 \\
1 / 4 & 1 / 3 & 1 / 2 & 1
\end{array}\right]
$$

By calculating the values of the relative weight, we get the eigenvector $W_{A}=(0.147,0.072,0.083$, 0.355), For the aim layer A, the maximum Eigenvalue $\lambda \max =4.2605, \mathrm{CI}=0.087, \mathrm{RI}=0.89$, and the relevant $\mathrm{CR}=0.0976<0.1$, thus high acceptability in the consistency of the judgment matrix. And for Guideline layer $B$, by using expert method, we can also get the relative matrix and the eigenvector. For layer $B, W_{B 1}=(0.643,0.074,0.283), W_{B 2}=(0.750,0.250), W_{B 3}=(0.667,0.333)$, and comparing judgment matrixes are as follows.

$$
B_{1}=\left[\begin{array}{llll}
1 & 2 & 2 & 4 \\
1 / 4 & 1 & 1 & 1 / 3 \\
1 & 1 / 2 & 1 & 1 / 2 \\
1 / 2 & 1 / 3 & 1 / 2 & 1
\end{array}\right] \quad B_{2}=\left[\begin{array}{cccc}
1 & 5 & 3 & 1 \\
1 / 5 & 1 & 1 / 3 & 1 \\
1 / 5 & 1 / 3 & 1 & 1 / 3 \\
1 / 5 & 3 & 1 & 1
\end{array}\right] \quad B_{3}=\left[\begin{array}{cccc}
1 & 3 & 3 & 1 \\
1 / 3 & 1 & 1 / 3 & 1 \\
1 / 3 & 1 / 3 & 1 & 1 / 3 \\
1 / 3 & 3 & 1 & 1
\end{array}\right] \quad B_{4}=\left[\begin{array}{cc}
1 & 8 \\
1 / 8 & 1
\end{array}\right]
$$

The relevant indicators of the above matrices are $\lambda \max =3.0940, \mathrm{CI}=0.047, \mathrm{RI}=0.052$, and the relevant $\mathrm{CR}=0.0904<0.1 ; \lambda \max =2.0000, \mathrm{CI}=0, \mathrm{RI}=0$, and the relevant $\mathrm{CR}=0<0.1 ; \lambda \max =3.0324$, $\mathrm{CI}=0.0162, \mathrm{RI}=0.52$, and the relevant $\mathrm{CR}=0.0311<0.1 ; \lambda \max =5.4313, \mathrm{CI}=0.0108, \mathrm{RI}=1.12$, and the relevant $\mathrm{CR}=0.0963<0.1$, respectively, and suggestes that the evaluation system has a satisfactory consistency.

Empirical Analysis. According to the comparing judgment matrixes, the values of the relative weight are calculated, and the coefficients of values of the relative weight of all indexes are also computed layer by layer. After getting the relative weight of each layer, the relative weight of each evaluation index is obtained. Because the evaluation system is very complicated and some of the indexes are difficult or impossible to be quantified, expert method is used to decide the value of grade $\left(V_{i}\right)$. Each index is divided into 5 grades from 1 to 5 . The higher the value is, the better the situation of index is. Twenty experts are chosen to give the value of each index and weighted average is calculated to determine the value. The weight of each layer and the values of evaluated indexes are listed as

The extent of OBE postgraduate education quality evaluation system construction is calculated by the following equation:

$$
G=\sum_{i=1}^{14} E_{i} \times V_{i}
$$

The extent of integration is assessed: from 0 to 1 , the integration degree is very bad; from 1 to 2 , the degree is not good; from 2 to 3, the degree is normal; from 3 to 4 , the degree is good; from 4 to 5, the degree is extremely good.

According to Eq. 5 and the data in table 1, we get the following evaluation degree:

$$
G=\left(E_{1}, E_{2}, E_{3}, \cdots, E_{14}\right) \times\left(V_{1}, V_{2}, V_{3}, \cdots, V_{14}\right)^{T}=3.995
$$

The result shows that the integration situation for OBE construction for postgraduate education quality evaluation system in China is normal and in good reliability. 
Table 3 Weights and values of all evaluation indexes of OBE postgraduate education quality system

\begin{tabular}{|c|c|c|c|}
\hline Index $D_{i}$ & Eigenvector $W_{i}$ & Weight $E_{i}$ & Value $V_{i}$ \\
\hline D1 & 0.033 & 0.031 & 2.1 \\
D2 & 0.327 & 0.073 & 2 \\
D3 & 0.032 & 0.001 & 2 \\
D4 & 0.159 & 0.002 & 2.3 \\
D5 & 0.217 & 0.005 & 2.1 \\
D6 & 0.302 & 0.03 & 2.2 \\
D7 & 0.414 & 0.004 & 2.1 \\
D8 & 0.183 & 0.025 & 1.1 \\
D9 & 0.308 & 0.007 & 2.2 \\
D10 & 0.105 & 0.003 & 2.1 \\
D11 & 0.211 & 0.002 & 1.3 \\
D12 & 0.068 & 0.007 & 3.2 \\
D13 & 0.242 & & 2.2 \\
D14 & 0.312 & \\
\hline
\end{tabular}

\section{Conclusions}

Comprehensive evaluation on OBE construction in postgraduate education quality evaluation system in China is a complex and important research issues. Fuzzy comprehensive evaluation method is a flexible evaluation method which can be effectively used in comprehensive evaluation on OBE construction for postgraduate education quality evaluation system. This paper uses AHP- fuzzy mathematics to establish an index system to evaluation the integration degree of OBE construction for postgraduate education quality evaluation system, and utilizes the index system and evaluation model to compute the evaluation results.

\section{Acknowledgements}

This work was supported by Postgraduate Teaching Funds for "On Outcome-Based Postgraduate Education Quality Evaluation System" by Northwest University for Nationalities.

\section{References}

[1] Xiong Ling, The current problem of graduate education in our country and improvement countermeasures. Journal of Henan industrial University journal. 2 (2010) 107-112.

[2] Tung Ting, The China and UK postgraduate education external quality assurance of analysis and reference. Journal of college education. 1 (2012)78-79.

[3] Pan Wuling, Graduate education in our country development present situation and existing problems. Journal of Lanzhou Institute of Education. 4(2011)11-12.

[4] Cascio, Costing Human Resources: The Financial Impact of Behavior in Organization. California Management Review. 48(2006)41-59.

[5] Anthoy Smith and drank Webster, The Postmodem University Contest Visions of Higher Education in Society. Buckingham. Open University Press. 1997.

[6] Cave M. Hanney S, In the Encyclopedia of Higher Education. Pergamon Press Ltd.1992.

[7] Jens-Christian Smeby, Disciplinary Differences in Graduate Education in Norway, Studies in Higher Education. 2012. 